\title{
SINGULAR INTEGRALS IN PRODUCT DOMAINS AND THE METHOD OF ROTATIONS
}

\author{
DONALD KRUG
}

(Communicated by Richard R. Goldberg)

\begin{abstract}
Singular integrals with kernels of the form $K(x, y)$ where $K$ satisfies conditions to be a bounded singular integral operator in each of its variables have been much studied lately. In this paper we use the classical method of rotations to give a proof that kernels of the form $K(x, y)=\Omega(x, y) /|x|^{n}|y|^{m}$ correspond to bounded singular integral operators.
\end{abstract}

The purpose of this paper is to use the method of rotations to give a simple proof that Calderón-Zygmund type operators when generalized to product domains are bounded operators. In particular we consider kernels of the type

$$
K(x, y)=\frac{\Omega(x, y)}{|x|^{n}|y|^{m}}
$$

for $x \in \mathbf{R}^{n}, y \in \mathbf{R}^{m}$ and $\Omega$ satisfying certain conditions (which make it a $\mathrm{C}-\mathrm{Z}$ kernel in each variable.) We are asking if $\|K * f\|_{p} \leq C_{p}\|f\|_{p}$.

If $\Omega(x, y)=\Omega_{1}(x) \Omega_{2}(y)$ where $\Omega_{1}$ and $\Omega_{2}$ correspond to bounded operators on $L^{p}$ then we can simply iterate one variable methods. In the case above this approach does not work. Kernels $K(x, y)$ not of the form of (1) but satisfying size and smoothness conditions like those of $1 / x y$ have been much studied lately (see $[2, \mathbf{3}])$. The kernels we will study in this paper are less general but can be handled entirely with single variable methods.

Before proceeding I want to thank Alberto Torchinsky for suggesting this approach.

We will proceed to use the method of rotations by studying even and odd kernels.

THEOREM 1. Let $K(x, y)=\Omega(x, y) /|x|^{n}|y|^{m}, \Omega$ odd in both variables, homogeneous of degree zero and $\int_{\Sigma_{n-1}} \int_{\Sigma_{m-1}}\left|\Omega\left(x^{\prime}, y^{\prime}\right)\right| d x^{\prime} d y^{\prime}<\infty$. (Here $\Sigma_{n-1}$ denotes the unit sphere in $\mathbf{R}^{n}$ and $x^{\prime}=x /|x|$.)

If $T_{\varepsilon, \eta}(f)(x, y)=\int_{|s|>\varepsilon} \int_{|t|>\eta} f(x-s, y-t) K(s, t) d s d t$ then

$$
\left\|\sup _{\varepsilon, \eta}\left|T_{\varepsilon, \eta}(f)\right|\right\|_{p} \leq C_{p}\|f\|_{p}, \quad 1<p<\infty .
$$

PROOF. Using polar coordinates, let $s=r_{1} s^{\prime}, t=r_{2} t^{\prime}$, then

$$
\begin{aligned}
K_{\varepsilon, \eta} & * f(x, y)=\int_{\Sigma_{n-1}} \int_{\Sigma_{m-1}} \Omega\left(s^{\prime}, t^{\prime}\right) \int_{\varepsilon}^{\infty} \int_{\eta}^{\infty} \frac{f\left(x-r_{1} s^{\prime}, y-r_{2} t^{\prime}\right)}{r_{1} r_{2}} d r_{1} d r_{2} d s^{\prime} d t^{\prime} \\
& =-\int_{\Sigma_{n-1}} \int_{\Sigma_{m-1}} \Omega\left(s^{\prime}, t^{\prime}\right) \int_{\varepsilon}^{\infty} \int_{\eta}^{\infty} \frac{f\left(x+r_{1} s^{\prime}, y-r_{2} t^{\prime}\right)}{r_{1} r_{2}} d r_{1} d r_{2} d s^{\prime} d t^{\prime}
\end{aligned}
$$

Received by the editors July 10, 1987.

1980 Mathematics Subject Classification (1985 Revision). Primary 42B20. 
since $\Omega$ is odd in the first variable. So the above expression equals

$$
\begin{aligned}
& \frac{1}{2} \int_{\Sigma_{n-1}} \int_{\Sigma_{m-1}} \Omega\left(s^{\prime}, t^{\prime}\right) \\
& \quad \times \int_{\varepsilon}^{\infty} \int_{\eta}^{\infty} \frac{f\left(x-r_{1} s^{\prime}, y-r_{2} t^{\prime}\right)-f\left(x+r_{1} s^{\prime}, y-r_{2} t^{\prime}\right)}{r_{1} r_{2}} d r_{1} d r_{2} d s^{\prime} d t^{\prime} \\
& =\frac{1}{2} \int_{\Sigma_{n-1}} \int_{\Sigma_{m-1}}^{\infty} \Omega\left(s^{\prime}, t^{\prime}\right) \int_{\left|r_{1}\right|>\varepsilon} \int_{\eta}^{\infty} \frac{f\left(x-r_{1} s^{\prime}, y-r_{2} t^{\prime}\right)}{r_{1} r_{2}} d r_{1} d r_{2} d s^{\prime} d t^{\prime}
\end{aligned}
$$

Doing the same in the second variable we obtain

$$
\frac{1}{4} \int_{\Sigma_{n-1}} \int_{\Sigma_{m-1}} \Omega\left(s^{\prime}, t^{\prime}\right) \int_{\left|r_{1}\right|>\varepsilon} \int_{\left|r_{2}\right|>\eta} \frac{f\left(x-r_{1} s^{\prime}, y-r_{2} t^{\prime}\right)}{r_{1} r_{2}} d r_{1} d r_{2} d s^{\prime} d t^{\prime} .
$$

Let $S$ be the hyperplane perpendicular to $s^{\prime}$, and $T$ to $t^{\prime}$. Let $x=z+\lambda s^{\prime}$ with $z \in S, y=w+\mu t^{\prime}$ with $w \in T$. Then

$$
\begin{aligned}
= & \frac{1}{4} \int_{\Sigma_{n-1}} \int_{\Sigma_{m-1}} \Omega\left(s^{\prime}, t^{\prime}\right) \\
& \times \int_{\left|r_{1}\right|>\varepsilon} \frac{1}{r_{1}}\left(\int_{\left|r_{2}\right|>\eta} \frac{f\left(z+(\lambda-r) s^{\prime}, w+(\mu-r) t^{\prime}\right)}{r_{2}} d r_{2}\right) d r_{1} d s^{\prime} d t^{\prime} .
\end{aligned}
$$

So

$$
\begin{aligned}
& \left\|\sup _{\varepsilon, \eta}\left|T_{\varepsilon, \eta}(f)\right|\right\|_{p} \leq \int_{\Sigma_{n-1}} \int_{\Sigma_{m-1}}\left|\Omega\left(s^{\prime}, t^{\prime}\right)\right| \\
& \times\left(\int_{T} \int_{S} \int_{\mathbf{R}} \int_{\mathbf{R}} \sup _{\varepsilon>0} \mid \int_{\left|r_{1}\right|>\varepsilon} \frac{1}{r_{1}}\right. \\
& \left.\quad \times\left(\sup _{\eta>0} \mid \int_{\left|r_{2}\right|>\eta} \frac{f\left(z+\left(\lambda-r_{1}\right) s^{\prime}, w+\left(\mu-r_{2}\right) t^{\prime}\right)}{r_{2}}\right) d r_{2}\left|d r_{1}\right|^{p} d \lambda d \mu d w d z\right)^{1 / p} d s^{\prime} d t^{\prime} \\
& \leq \frac{C_{p}}{4} \int_{\Sigma_{n-1}} \int_{\Sigma_{m-1}}\left|\Omega\left(s^{\prime}, t^{\prime}\right)\right| d s^{\prime} d t^{\prime}\|f\|_{p} \leq C_{p}\|f\|_{p},
\end{aligned}
$$

using the boundedness of the Maximal Hilbert transform twice.

Exploiting this method we can in fact obtain

THEOREM 2. Let $K(x, y)=\Omega\left(x^{\prime}, y^{\prime}\right) /|x|^{n}|y|^{m}$, where $\Omega$ is odd in the $x^{\prime}$ variable. Let $K_{x}(y)=K(x, y)$ and $T_{\varepsilon}^{x}(f)(y)=\int_{|t|>\varepsilon} f(x, y-t) K_{x}(t) d t$. If

$$
\left\|\sup \left|T_{\varepsilon}^{x}(f)\right|\right\|_{p} \leq C_{p}\|f\|_{p}
$$

$C_{p}$ independent of $x$ (i.e., $K$ is a bounded $C$ - $Z$ kernel in $y$ independent of $x$ ), then

$$
\left\|\sup _{\varepsilon, \eta}\left|T_{\varepsilon, \eta}(f)\right|\right\|_{p} \leq C_{p}\|f\|_{p}
$$

ProOF. Proceeding as above, but in the $x$-variable only,

$$
K_{\varepsilon, \eta} * f(x, y)=\frac{1}{2} \int_{\Sigma_{n-1}} \int_{|t|>\eta} \frac{\Omega\left(s^{\prime}, t\right)}{|t|^{m}} \int_{|r|>\varepsilon} \frac{f\left(x-r s^{\prime}, y-t\right)}{r} d r d t d s^{\prime} .
$$


Let $x=z+\lambda s^{\prime}, x \in S$, where $S$ is perpendicular to $s^{\prime}$,

$$
=\frac{1}{2} \int_{\Sigma_{n-1}} \int_{|t|>\eta} \frac{\Omega\left(s^{\prime}, t\right)}{|t|^{m}} \int_{|r|>\varepsilon} \frac{f\left(z-(\lambda-r) s^{\prime}, y-t\right)}{r} d r d t d s^{\prime} .
$$

So

$$
\begin{aligned}
&\left\|\sup _{\varepsilon, \eta}\left|T_{\varepsilon, \eta}\right|\right\|_{p} \\
& \leq \frac{1}{2} \int_{\Sigma_{n-1}}\left(\int_{S} \int_{\mathbf{R}} \int_{\mathbf{R}^{m}} \sup _{\eta>0} \mid \int_{|t|>\eta} \frac{\Omega\left(s^{\prime}, t\right)}{t}\right. \\
&\left.\quad \times\left.\sup _{\varepsilon>0}\left|\int_{|r|>\varepsilon} \frac{f\left(z-(\lambda-r) s^{\prime}, y-t\right)}{r} d r\right| d t\right|^{p} d y d \lambda d z\right)^{1 / p} d s^{\prime} .
\end{aligned}
$$

Now using the assumption $(*)$, that our operator is bounded as an operator acting only in the second variable, we have

$$
\begin{aligned}
& \leq C_{p} \int_{\Sigma_{n-1}}\left(\int_{\mathbf{R}^{m}} \int_{S} \int_{\mathbf{R}} \sup _{\varepsilon>0}\left|\int_{|r|>\varepsilon} \frac{f\left(z-(\lambda-r) s^{\prime}, y\right)}{r} d r\right|^{p} d \lambda d z d y\right)^{1 / p} d s^{\prime} \\
& \leq C_{p} \int_{\Sigma_{n-1}}\left(\int_{\mathbf{R}^{m}} \int_{S} \int_{\mathbf{R}}|f(x, y)|^{p} d x d y\right)^{1 / p} d s^{\prime}=C_{p}\left(\int_{\Sigma_{n-1}} d s^{\prime}\right)\|f\|_{p} .
\end{aligned}
$$

THEOREM 3. Again let $K(x, y)=\Omega(x, y) /|x|^{n}|y|^{m}, \Omega$ homogeneous of degree zero in each variable, $\int_{\Sigma_{n-1}} \Omega(s, t) d s=0$ a.e. in $t, \int_{\Sigma_{m-1}} \Omega(s, t) d t=0$ a.e. in $s,\left(\int_{\Sigma_{n-1}}|\Omega(s, t)|^{2} d s\right)^{1 / 2}<C$ independent of $t$, and $\left(\int_{\Sigma_{m-1}}|\Omega(s, t)|^{2} d t\right)^{1 / 2}<C$ independent of $s$. Then $\|K * f\|_{p} \leq C_{p}\|f\|_{p}$.

PROOF. We may assume $\Omega$ is even in both variables (since the hypotheses assure that both the previous theorems hold) and that $f=\rho$, a testing function. Let $y$ be fixed, $K_{y}(x)=\Omega\left(x^{\prime}, y^{\prime}\right) /|x|^{n}, T_{y}(\rho)=K_{y} * \rho$ and $R_{i}$ be the $i$ th Riesz Transformation in $x$. Then $[4, \mathrm{p} .225]$ shows that $R_{i} T_{y}$ is essentially an odd C-Z operator. In fact, it is shown that $\left(R_{i} T_{y}\right)^{\wedge}=\left(J_{i}^{y}\right)^{\wedge}$ where $J_{i}^{y}(x)=\omega_{y^{\prime}}\left(x^{\prime}\right) /|x|^{n}$, $\omega \in L^{2}\left(\Sigma_{n-1}, d x\right)$ and $\omega_{y}(x)$ is odd in $x$.

If we set

$$
J_{i}(x, y)=\frac{J_{i}^{y}(x)}{|y|^{m}}=\frac{\omega\left(x^{\prime}, y^{\prime}\right)}{|x|^{n}|y|^{m}}, \quad \text { where } \omega\left(x^{\prime}, y^{\prime}\right)=\omega_{y^{\prime}}\left(x^{\prime}\right)
$$

then $J_{i}$ is an odd C-Z integral operator in the $x$-variable.

In the $y$-variable we see that $J_{i}$ is a C-Z operator (as expected) since

$$
\left[\left(R_{j} T\right) \rho\right]^{\wedge}=-i \frac{x_{j}}{|x|} \Omega_{0}\left(\frac{x}{|x|}, \frac{y}{|y|}\right) \hat{\rho}
$$

where $\Omega_{0}=\widehat{K}$ (see [4]). In the $y$-variable this acts exactly as did $K * \rho$ (up to a constant $C_{x},\left|C_{x}\right|<1$ ) and so is still a $\mathrm{C}-\mathrm{Z}$ operator.

So by Theorem 2 above, $\left\|J_{i} * \rho\right\|_{p} \leq C\|\rho\|_{p}$.

It follows that

$$
\|K * \rho\|_{p}=\left\|\Sigma R_{i} * J_{i} * \rho\right\|_{p} \leq \sum\left\|R_{i} *\left(J_{i} * \rho\right)\right\|_{p} \leq C\|\rho\|_{p}
$$


There is a weighted version of this as well.

For one variable $x \in \mathbf{R}^{n}$, a function $w(x)>0$ is called an $A_{p}$-weight, $1<p<\infty$, if it satisfies

$$
\frac{1}{|Q|} \int_{Q} w(x) d x\left(\frac{1}{|Q|} \int_{Q}[w(x)]^{-1 /(p-1)} d x\right)^{p-1} \leq B
$$

for some $B<\infty$ and all cubes $Q$ in $\mathbf{R}^{n}$.

The following theorem is well known.

THEOREM. If $w$ is an $A_{p}$-weight and $K$ is a standard $C-Z$ kernel then

$$
\int_{\mathbf{R}^{n}}|f * K(y)|^{p} w(y) d y \leq C(p, B) \int_{\mathbf{R}^{n}}|f(y)|^{p} w(y) d y .
$$

See [1] for results on weights.

In the work above we used only that $T=K * f$ was bounded in $L^{p}$-norm in each variable separately. Thus if $w(x, y)$ is an $A_{p}$-weight in each variable, i.e., if $w(x, y)>0, x \rightarrow w(x, y)$ is an $A_{p}$-weight with $B$ independent of $y$ and similarly for $y \rightarrow w(x, y)$ then following the method of proof above we have the following.

THEOREM 4. If $w$ is as above and $K$ satisfies the conditions of Theorem 3 then

$$
\left(\int_{\mathbf{R}^{n} \times \mathbf{R}^{m}}|K * f(y)|^{p} w(y) d y\right)^{1 / p} \leq C(p, A)\left(\int_{\mathbf{R}^{n} \times \mathbf{R}^{m}}|f(y)|^{p} w(y) d y\right)^{1 / p} .
$$

\section{REFERENCES}

1. R. Coifman and C. Fefferman, Weighted norm inequalities for maximal functions and singular integrals, Studia Math. 51 (1974), 241-250.

2. R. Fefferman, Singular integrals on product $H^{p}$-spaces, preprint.

3. R. Fefferman and E. M. Stein, Singular integrals on product spaces, Adv. in Math. 45 (1982), 117-143.

4. E. M. Stein and G. Weiss, Introduction to Fourier analysis on Euclidean spaces, Princeton Univ. Press, Princeton, N. J., 1971.

Department of MAthematical Sciences, NORThern KentuCKy UNiversity, HIGHLAND HEIGHTS, KENTUCKY 41076 